\title{
Solvable and Nilpotent Structures
}

By

\author{
Akito TsuBoI*
}

\section{§ 0. Introduction}

In [6], we have proven that every non- $\aleph_{1}$-categorical connected nilpotent group of finite Morley rank has the center $Z(G)$ with $\operatorname{RM}(Z(G)) \geq 2$. If a connected nilpoten group has a finite Morley rank, then it is easy to see that the center is infinite. So the above result shows if $G$ is non- $\boldsymbol{\aleph}_{1}$-categorical the center has a rather complicated structure. The present paper is a natural continuation of [6]. In this paper we introduce the notion of $*$-nilpotency, and prove *-versions of results in [6]. (See Theorems 2.3, and 2.6.) Since our setting is rather general, we can apply our results to $\omega$-stable ring theory. (See Corollary 2.5.)

\section{§1. Preliminaries}

The reader is supposed to have enough knowledge of stable model theory and stable group theory. Especially we assume the reader is accustomed to handling the independence relation in the sense of non-forking. Throughout $G$ is an $\omega$-stable structure including the group structure $\left(G,{ }^{\circ}\right)$. We assume $G$ is sufficiently saturated. (This assumption is trivial, since we can extend $G$ to a model with a sufficiently large saturation.) If $A$ is a subset of $G,\langle A\rangle$ denotes the group generated by $A . \quad \operatorname{RM}(\varphi)$ is the Moley rank of a formula $\varphi$. A type $p \in \mathrm{S}(A)$ is called a generic type if it has the maximum rank among the types in $\mathrm{S}(A)$ (i.e. $\mathrm{RM}(p)=\max \{\operatorname{RM}(q): q \in \mathrm{S}(A)\})$. We assume $\mathrm{RM}(G)$ is finite. A formula $\varphi$ is said to be strongly minimal if $\operatorname{RM}(\varphi)=1$ and $\operatorname{deg}(\varphi)=1$. We say simply a type $p$ is strongly regular if $p$ is stationary and strongly regular. It is well-known that an $\omega$-stable theory is $\aleph_{1}$-categorical if and only if it unidimensional. We use this fact freely.

Communicated by S. Takasu, March 16, 1988.

* Institute of Mathematics, University of Tsukuba, Tsukuba, Ibaraki 305, Japan. 
Our main tool is Zil'ber's Indecomposability Theorem in [7]. It states that if $A_{i} \subset G(i \in I)$ are indecomposable subsets containing 1 , then the group $\left\langle A_{i}\right\rangle_{i \in I}$ generated by $A_{i}$ 's is definable and connected. The fact that the indecomposability is preserved by endomorphism is also important.

\section{§ 2. Main Theorem}

In this section, we introduce the notion of $*$-solvability and $*$-nilpotency. We proves $*$-versions of the results in [6]. First we need the following definitions:

Definition 2.1. Let $x * y$ be a definable action on $G$. Moreover we assume each $a * x: \quad G \rightarrow G(a \in G)$ is an endomorphism of the group structure $(G, \bullet)$. Then we say:

(1) A subfroup $H \subset G$ is $*$-normal if $H$ is closed under all $a * x(a \in G)$;

(2) A subgroup $H \subset G$ is $*$-central if each $a * x(a \in G)$ is identical on $H$;

(3) A subgroup $H \subset G$ is $*$-trivial if each $a * x(a \in H)$ is identical on $H$;

(4) $G$ is *-solvable if there is a descending sequence $G=G_{0} \supset G_{1} \supset \cdots \supset G_{n}=\{1\}$ of subgroups of $G$ with $a * x=x\left(\bmod G_{i+1}\right)$ for all $a, x \in G_{i}$.

(5) $\mathrm{G}$ is *-nilpotent if there is a descending sequence $G=G_{0} \supset G_{1} \supset \cdots \supset G_{n}=$ $\{1\}$ of subgroups of $G$ with $a * x=x\left(\bmod G_{i+1}\right)$ for all $a \in G$, and $x \in G_{i}$.

The following lemma is a generalization of Zil'ber's theorem which guarantees the indecomposability of $[X, H]$ for any indecomposable $H$. The proof of Lemma 2.2 goes almost pararell with that of Zil'ber.

Lemma 2.2. Let $E$ be a set of endomorphisms of $G$ and $H$ an indecomposable subset of $G$. Then the group generated by:

$$
C=\left\{f(a) \cdot a^{-1}: f \in E, a \in H\right\}
$$

is indecomposable (connected).

Proof. It is sufficient to prove the lemma for $E=\{f\}$. By way of a contradiction, suppose that $C$ is not indecomposable. Then there are $a_{1}, \cdots, a_{n} \in H$ and a definable group $N \subset G$ such that

$$
C \subset f\left(a_{1}\right) \cdot a_{1}^{-1} \cdot N \cup \cdots \cup f\left(a_{n}\right) \cdot a_{n}^{-1} \cdot N .
$$

Suppose that $n$ is chosen to be minimal. Let $g \in G$ be an arbitrary element. Since $f(g) \cdot C \cdot g^{-1}=C$, we have

$$
(*)_{g}: \quad C \subset f\left(g \cdot a_{1}\right) \cdot\left(g \cdot a_{1}\right)^{-1} \cdot g \cdot N \cdot g^{-1} \cup \cdots \cup f\left(g \cdot a_{1}\right) \cdot\left(g \cdot a_{1}\right)^{-1} \cdot g \cdot N \cdot g^{-1} \text {. }
$$


Note that each $g \cdot N \cdot g^{-1}$ is a definable group. Hence $N_{0}=\bigcap\left\{g \cdot N \cdot g^{-1}: g \in G\right\}$ is in fact a finite intersection of $g \cdot N \cdot g^{-1}$ 's. By intersecting all the right hand sides of $(*)_{g}(g \in G)$, we have

$$
C \subset d_{1} \cdot N_{0} \cup \cdots \cup d_{n} \cdot N_{0}
$$

for some $d_{1}, \cdots, d_{n} \in C$.

Again, by multiplying $f(g)$ from the left and $g$ from the left, we have

$$
C \subset f(g) \cdot d_{1} \cdot g^{-1} \cdot g \cdot N_{0} \cdot g^{-1} \cup \cdots \cup f(g) \cdot d_{n} \cdot g^{-1} \cdot g \cdot N_{0} \cdot g^{-1} .
$$

Since $N_{0}$ is a normal subgroup of $G$, we have

$$
C \subset f(g) \cdot d_{1} \cdot g^{-1} \cdot N_{0} \cup \cdots \cup f(g) \cdot d_{n} \cdot g^{-1} \cdot N_{0} .
$$

By the minimlity of $n$, for each $i<\omega$, there is $j<\omega$ such that

$$
f(g) \cdot d_{i} \cdot g^{-1} \cdot N_{0}=d_{j} \cdot N_{0} .
$$

Thus each $f(g) \cdot x \cdot g^{-1}(g \in G)$ acts trivially on the set $\left\{d_{1} \cdot N_{0}, \cdots, d_{n} \cdot N_{0}\right\}$. Let $f(h) \cdot h^{-1}$ be an arbitrary element of $C$. Choose $e_{1}$ so that $d_{1}=f\left(e_{1}\right) \cdot e_{1}^{-1}$. Then

$$
\begin{aligned}
f(h) \cdot h^{-1} & =f\left(h \cdot e_{1}^{-1} \cdot e_{1}\right) \cdot\left(h \cdot e_{1}^{-1} \cdot e_{1}\right)^{-1} \\
& =f\left(h \cdot e_{1}^{-1}\right) \cdot\left[f\left(e_{1}\right) \cdot e_{1}^{-1}\right] \cdot\left(h \cdot e_{1}^{-1}\right)^{-1} \\
& =f\left(h \cdot e_{1}^{-1}\right) \cdot d_{1} \cdot\left(h \cdot e_{1}^{-1}\right)^{-1} \\
& =d_{1}\left(\bmod N_{0}\right) .
\end{aligned}
$$

The last line follows from the triviality of the action. Hence $f(h) \cdot h^{-1} \in d_{1} \cdot N_{0}$. Hence $C \subset d_{1} \cdot N_{0}$ and so $C$ is indecomposable.

Theorem 2.3. Suppose that $G$ is an *-nilpotent group of finite Morley rank. Then:

(1) $G$ is $\aleph_{1}$-categorical; or

(2) There is a definable *-central subgroup $G^{*}$ of $G$ with $\operatorname{RM}\left(G^{*}\right) \geq 2$

Proof. We can assume $G$ is connected. First we work under the assumption that there are two mutually orthogonal strongly minimal types $p$ and $q$. By adding constants to the language we can assume $p$ and $q$ are pure types. Choose strongly minimal and indecomposable formulas $\delta \in p$ and $\varepsilon \in q$. Choose $a \in \delta$ and $b \in \varepsilon$ arbitrarily and let $N$ and $H$ be the $*$-normal closures of $a^{-1} \cdot \delta$ and $b^{-1} \cdot \varepsilon$ respectively. More precisely, let:

$$
N=\left\langle g *\left(a^{-1} \cdot \delta\right): g \in G\right\rangle ; H=\left\langle g *\left(b^{-1} \cdot \varepsilon\right): g \in G\right\rangle .
$$

By the indecomposability theorem we know that $N$ and $H$ are definable. The 
orthogonality of $p$ and $q$ implies that $N \cap H$ is finite. We construct two sequences $\left(N_{i}\right)$ of subgrous of $N$ and $\left(H_{i}\right)$ of subgroups of $H$ by:

$$
\begin{aligned}
& N_{0}=N ; H_{0}=H ; \\
& N_{i+1}=\left\langle(a * b) \cdot b^{-1}: a \in G, b \in N_{i}\right\rangle ; \\
& H_{i+1}=\left\langle(a * b) \cdot b^{-1}: a \in G, b \in H_{i}\right\rangle .
\end{aligned}
$$

If $N_{i}$ is in $G_{j}$, then $N_{i+1}$ is in $G_{j+1}$. Thus there are $N_{i}$ and $H_{j}$ such that:

$$
\begin{aligned}
& N_{i} \neq\{1\} ; H_{j} \neq\{1\} ; \\
& N_{i+1}=H_{j+1}=\{1\} .
\end{aligned}
$$

By Lemma 2.2 above, $N_{k}$ and $H_{k}$ are all indecomposable, hence $N_{i}$ and $H_{j}$ above are infinite *-central groups. Since $N_{i} \cap H_{j} \subset N \cap H$ is a finite group, $G^{*}=$ $\left\langle N_{i}, H_{j}\right\rangle$ clearly has the Morley rank $\geq 2$. It is also clear that $G^{*}$ is $*$-central. Thus we have proven that (2) holds under the assumption that there are two mutually orthogonal strongly minimal types. We now assume that there is only one strongly minimal type modulo orthogonality. Let $p=\operatorname{tp}(b / A) \in S(A)$ be a regular type and $r \in \mathrm{S}(A \cup\{b\})$ a generic type. Let $I=\left(a_{i}\right)_{i<\omega}$ be a Morley sequence of $r$. We define the sequences $\left(b_{i}\right)_{i<\omega} \subset G$, and $\left(p_{i}\right)_{i<\omega} \subset \mathbb{S}(A I)$ by:

$$
\begin{aligned}
& b_{0}=b ; \\
& b_{i+1}=\left(a_{i} * b_{i}\right) \cdot b_{i}^{-1} \\
& p_{i}=\operatorname{tp}\left(b_{i} / A I\right) .
\end{aligned}
$$

If for every regular $p$ there is $p_{n}$ with $\operatorname{RM}\left(p_{n}\right)=1$, then $G$ is clearly $\aleph_{1}$-categorical. So we assume there is $p_{n}$ with $\operatorname{RM}\left(p_{n}\right) \geq 2$ and $\operatorname{RM}\left(p_{n+1}\right)=0$. Then:

$$
\begin{aligned}
& \left(a_{n} * b_{n}\right) \cdot b_{n}^{-1} \in \operatorname{acl}(A I), \text { hence } \\
& \left(a_{n} * b_{n}\right) \cdot b_{n}^{-1} \in \operatorname{acl}\left(A \cup\left\{a_{0}, \cdots, a_{n-1}, a_{n}\right\}\right) .
\end{aligned}
$$

Moreover $a_{n}$ and $b_{n}$ are independent over $A \cup\left\{a_{0}, \cdots, a_{n-1}\right\}$. Hence,

$$
(a * d) \cdot d^{-1} \in \operatorname{acl}\left(A \cup\left\{a_{0}, \cdots, a_{n-1}\right\} \cup\{a\}\right),
$$

whenever $a^{\wedge} d$ realizes the type $r\left|\left(A \cup\left\{a_{0}, \cdots, a_{n-1}\right\}\right) \otimes p_{n}\right|\left(A \cup\left\{a_{0}, \cdots, a_{n-1}\right\}\right)$. Let $a^{\wedge} d^{\wedge} e$ realize the type $r\left|\left(A \cup\left\{a_{0}, \cdots, a_{n-1}\right\}\right) \otimes p_{n}^{(2)}\right|\left(A \cup\left\{a_{0}, \cdots, a_{n-1}\right\}\right)$. Then we have

$$
\begin{aligned}
& (a * d) \cdot d^{-1}=(a * e) \cdot e^{-1}, \text { hence } \\
& \left(a *\left(d^{-1} \cdot e\right)\right)=d^{-1} \cdot e
\end{aligned}
$$

Let $C$ be the set $\left\{x \in G:\left(x *\left(d^{-1} \cdot e\right)\right)=d^{-1} \bullet e\right\}$. As $*$ is an action, $C$ is a 
definable group. Since $C$ contains a generic element a over $d^{-1} \cdot e$, by the connectedness of $G$, we have $C=G$. Hence if we set $G^{*}=\{x \in G: a * x=x$, for all $a \in G\} G^{*}$ is an *-central group. Moreover it contains $d^{-1} \cdot e$, hence $\operatorname{RM}\left(G^{*}\right) \geq \operatorname{RM}\left(d^{-1} \cdot e\right) \geq 2$. Thus (2) holds in this case.

We have the following corollaries:

Corollary 2.4. Let $G$ be a connected nilpotent group of finite Morley rank. Then $G$ is $\aleph_{1}$-categorical if $\operatorname{RM}(Z(G))=1$.

Proof. Define $a * b=a^{-1} \cdot b \cdot a$. Then $G$ is nilpotent if and only if it is *-nilpotent. Note that each $G^{n}=\left[G, G^{n-1}\right](n<\omega)$ is connected. If $G^{n} \neq\{1\}$ and $G^{n+1}=\{1\}$, then $G^{n}$ is an infinite group included in the center $Z(G)$. By Theorem 2.3, $\operatorname{RM}(Z(G)) \geq 2$, unless $G$ is $\aleph_{1}$-categorical. Hence $\operatorname{RM}(Z(G))=1$ implies that $G$ is $\aleph_{1}$-categorical.

In the proof of Theorem 2.3, the condition that $*$ is an action was necessary only for proving that the set $C_{a}=\{x: x * a=a\}$ is a group. Thus we have:

Corollary 2.5. Let $R$ be a non- $\aleph_{1}$-categorical connected ring of finite Morley rank. If $R$ is nilpotent $\left(R^{n}=R \cdots R=(0)\right)$, then there is an ideal $I$ of $R$ such that $\operatorname{RM}(I) \geq 2$ and $R \cdot I=0$.

Proof. For $a, b \in R$, let $a * b=a \cdot b+b$. Then each function $a * x$ of $x$ is an endomorphism of $R$. $(R,+)$ is $*$-nilpotent. Morever $a * b=b$ iff $a \cdot b=0$, hence $C_{a}=\{x \in R: x * a=a\}$ is a definable group. Now we can apply Theorem 2.3 to finish our proof.

Theorem 2.6. Suppose that $G$ is a *-solvable group of finite Morley rank. Then:

(1) $G$ is $\aleph_{1}$-categorical; or

(2) There is a definable *-trivial subgroup $G^{*}$ of $G$ with $\operatorname{RM}\left(G^{*}\right) \geq 2$;

Proof. First suppose that $G$ has two mutually orthogonal strongly minimal types. Under this supposition, we prove that the case (2) above holds. Let $N$ and $H$ be two infinite, connected, *-normal subgroups of $G$ with $\operatorname{acl}(N) \cap$ $\operatorname{acl}(H) \subset \operatorname{acl}(\phi)$. Moreover we can assume that $N$ and $H$ are $*$-trivial. W.l.o.g. we can assume $N$ and $H$ are strongly minimal. For showing $G^{*}=N \cdot H$ satisfies the condition in (2), it is sufficient to show:

(3) $(a * b)=b$, for all $a \in N$ and $b \in H$; and

(4) $(a * b)=b$, for all $a \in H$ and $b \in N$. 
By way of a contradiction, suppose that (3) is not the case. Let

$$
N_{0}=\{a \in N: \forall x \in H(x * a=a)\} .
$$

Clearly $N_{0}$ is a group. If $N_{0}$ is infinite, then $N_{0}=N$, so (3) holds. Hence $N_{0}$ is a finite group. For $a \in N-N_{0}$, let $H_{a}$ be the group defined by:

$$
H_{a}=\{x \in H: x * a=a\} \text {. }
$$

$H_{a}$ is also a finite group. Now choose a non-algebraic element $h \in H$. Let a be an arbitrary element in $N-N_{0}$ and set $b=h * a$. Then the set

$$
\{x \in H: x * a=b\}
$$

is included in $h \cdot H_{a}$ which is finite. Hence $h$ is algebraic over $\{a, b\} \subset N$. This is a contradiction, since we are assuming $\operatorname{acl}(N) \cap \operatorname{acl}(H) \subset \operatorname{acl}(\phi)$. Thus (3) (and (4)) holds, and we have a group of Morley rank 2 which is $*$-trivial.

Now consider the case when there is the unique generic type modulo orthogonality. The remainder of the present proof is almost identical with that of Theorem 2.3. So we give only a sketch. Let $I=\left(a_{i}\right)$ be a Morley sequence of a type $p$. Define:

$$
\begin{aligned}
& b_{i}^{0}=a_{i} \\
& b_{i}^{j+1}=\left(b_{2 i}^{j} * b_{2 i+1}^{j}\right) \cdot b_{2 i+1}^{j} .
\end{aligned}
$$

If every $p$ has $b_{i}^{j}$ with $\operatorname{RM}\left(b_{i}^{j}\right)=1$, then $G$ is unidimensional, hence $\aleph_{1}$-categorical. So suppose that there is a rank 2 type $p$ such that

$$
(a * b) \cdot b^{-1} \text { is algebraic, for every } a^{\wedge} b \text { realizing } p^{(2)} .
$$

Hence

$$
(a * b) \cdot b^{-1}=(d * b) \cdot b^{-1}, \text { for every } a^{\wedge} b^{\wedge} d \text { realizing } p^{(2)}
$$

Thus

$$
x * y=y \text {, for every } x^{\wedge} y \text { realizing } r,
$$

where $r$ is the type of $a \cdot b^{-1}$ for $a^{\wedge} b$ realizing $p^{(2)}$. (The Morley rank of $r$ is $\geq 2$.) Let $J$ be an infinite Morley sequence of $r$, and define:

$$
\begin{aligned}
& X=\{x \in G: \forall a \in I(x * a=a)\} ; \\
& Y=\{a \in X: \forall x \in X(x * a=a)\} .
\end{aligned}
$$

Then $Y$ is an $*$-trivial group of Morley rank $\geq 2$. 


\section{References}

[1] Kikyo, H., Some remarks on Zil'ber's results, to appear in Tsukuba J. of Math.

[2] Pillay, A., An Introduction to Stability Theory, Clarendon Press, Oxford (1983).

[3] Reineke, J., Minimal Gruppen, Z. Math Logik Grundlagen Math., 21 (1975), 357-379.

[4] Takahashi, M., Completeness of Boolean powers of Boolean algebras, J. of Math. Soc. Japan, 40 (1988), 445-456.

[5] Tanaka, K., Non-abelian groups of Morley rank 2, to appear in Math. Japonica.

[6] Tsuboi, A., On a property of $\omega$-stable solvable groups, to appear in Archive for Math. Log. (1989).

[7] Zil'ber, B.I., Groups and rings with categorical theories (in Russian), Fund. Math., 95 (1977), 173-188. 
